\title{
The Planetary Nebula Luminosity Function and its Issues
}

\author{
Robin Ciardullo \\ Department of Astronomy \& Astrophysics, Penn State University \\ 525 Davey Lab, University Park, PA \\ email: rbc@astro.psu.edu
}

\begin{abstract}
The bright-end cutoff of the [O III] $\lambda 5007$ planetary nebula luminosity function (PNLF) is insensitive to population age and metallicity, making it an excellent extragalactic standard candle. We review our knowledge of the function and discuss the challenges related to modeling it. We show that, while there has been a great deal of progress in understanding the nuances of its shape, there is still no solid theory which explains the luminosity of the PNLF cutoff in old stellar populations. This is an extremely serious problem, as it affects a myriad of astrophysical issues, from the determination of the stellar masses of galaxies to our understanding of alternative channels of stellar evolution.
\end{abstract}

Keywords. planetary nebulae: general, galaxies: distances and redshifts, stars: AGB and postAGB

\section{Introduction}

A little over 30 years ago, Ford (1983) noticed that the brightest planetary nebulae $(\mathrm{PN})$ in five, very different Local Group galaxies all emitted a similar amount of [O III] $\lambda 5007$ luminosity. Shortly thereafter, Ciardullo et al. (1989a,b), Jacoby et al. (1990a), and Hui et al. (1993) showed that, shockingly, the absolute luminosity of the bright-end cutoff of the [O III] $\lambda 5007$ planetary nebula luminosity function (PNLF) was the same (to within a few percent) for all early-type stellar populations. Jacoby et al. (1990b) and Feldmeier et al. (1997) later expanded that statement to include late-type galaxies (with the $[\mathrm{O} \mathrm{III}] / \mathrm{H} \alpha$ ratio used as a $\mathrm{PN} / \mathrm{H}$ II region discriminant) and since then, PNLF measurements have been performed in dozens of galaxies, both for connecting the Pop I and Pop II distance ladders, and a first step in the study of the stellar kinematics of galaxies (e.g., Méndez et al. 2001; Ciardullo et al. 2004; Teodorescu et al. 2010).

When the PNLF was first measured, the distance scale of the universe was uncertain to a factor of almost two, and a major goal of the era was to obtain a reasonably accurate distance to the Virgo Cluster. Today, in this era of precision cosmology, $10 \%$ distances are no longer thought of as interesting: one wants measurements that are better than $\sim 3 \%$. So, in this environment, what are the key issues for the PNLF and where should astronomers focus their attention?

\section{Just How Invariant is the PNLF Cutoff?}

Using their M31 observations as a guide, Ciardullo et al. (1989a) proposed that

$$
N(M) \propto e^{0.307 M}\left\{1-e^{3\left(M^{*}-M\right)}\right\} \quad \text { with } \quad M=-2.5 \log F_{5007}-13.74
$$

was a good analytical representation of PNLF. In this parameterization, $M^{*}$, the absolute [O III] magnitude of the brightest possible PN, is derived from the PNLFs of galaxies 
with Cepheid and Tip of the Red Giant Branch distances (Ciardullo et al. 2002; Ciardullo 2013). The key question of the 1990's was whether $M^{*}$ varies with stellar population.

Remarkably, despite numerous tests using subsamples of PN within galaxies (e.g., Hui et al. 1993; Ciardullo et al. 2004; Merrett et al. 2006), within the galaxies of a common group or cluster (e.g., Ciardullo et al. 1989b; McMillan et al. 1993; Jacoby et al. 1990a), and within galaxies with known distances (Ciardullo et al. 2002; Ciardullo 2013), the only observed systematic behavior is the expected fading of $M^{*}$ in low-metallicity populations (Dopita et al. 1992; Schönberner et al. 2010). In systems more metal-rich than the LMC, one derives $M^{*}=-4.53 \pm 0.05$ with amazing consistency (Ciardullo 2013). Since, the statistical uncertainty associated with a PNLF measurement to a large galaxy can be $\lesssim 5 \%$, this suggests that the technique may be precise enough for current cosmology.

Nevertheless, there are still some questions about the reliability of the PNLF technique. In their final summary of the HST Distance Scale Key Project, Ferrarese et al. (2000) and Freedman et al. (2001) excluded the PNLF from consideration, as they found that once beyond $\sim 10 \mathrm{Mpc}$, the PNLF seemed to produce distances that were 0.2 to $0.3 \mathrm{mag}$ smaller than those derived from Cepheids and the Surface Brightness Fluctuation (SBF) method. Ciardullo et al. (2002) showed that much of this offset was likely due to a small systematic difference between the internal extinction within the PNLF and SBF calibrating galaxies (i.e., mostly late-type spirals with Cepheid distances) and the distant elliptical and lenticular program galaxies. Specifically, the existence of $E(B-V) \sim 0.02$ of additional internal reddening in nearby spiral bulges would cause SBF distances to be overestimated by $0.08 \mathrm{mag}$, and PNLF distances to be underestimated by $0.07 \mathrm{mag}$. This goes a long way towards explaining $\sim 0.3$ mag offset between the two techniques.

But another issue which may affect the PNLF distance scale involves the population of PN progenitors. Recently, Badenes et al. (2015) correlated the positions of $435 \mathrm{PN}$ in the Large Magellanic Cloud with the distribution of stellar ages at each location in the galaxy. Their conclusion was that most PN progenitors have ages either between 5 and $8 \mathrm{Gyr}$ (i.e., main-sequence masses between $1.2>M / M_{\odot}>1.0$ ), or between $35<\tau<800 \mathrm{Myr}$ (progenitor masses between $8.2>M / M_{\odot}>2.1$ ). No signal was seen at any other age. Moreover, for the galaxy's brightest PN, only the older, lower-mass progenitors appear to be important. Small number statistics limit the robustness of this measurement, but if the result holds, it may indicate the existence of a small, counter-intuitive systematic error, with older stellar populations producing slightly brighter $(\sim 0.1 \mathrm{mag})$ planetaries.

\section{Beyond the Bright-End Cutoff}

One aspect of PNLF research that has received a great deal of attention is the modeling of the PNLF shape beyond the brightest $\sim 1 \mathrm{mag}$ of the luminosity function. In general terms, a planetary nebula can be divided into two components: a freely expanding lowdensity nebula, and a hot, evolving central star. If the timescale for nebular expansion dominates, i.e., $\tau_{\mathrm{Neb}} \ll \tau_{\mathrm{Star}}$, then the luminosity of the PNe will decline as

$$
L \propto N_{H} N_{e} V \propto R^{-3} \propto t^{-3} \Longrightarrow N(M) \propto e^{0.307 M}
$$

(Henize \& Westerlund 1963). On the other hand, if the timescale for central star evolution is more rapid than that of the nebula, then the luminosity function should be nonmonotonic, as post-AGB stars spend most of their time either at high-luminosity (in their last stages of shell burning), or as slowly cooling white dwarfs. The shape of the PNLF therefore encodes information about the relationship between these two timescales.

Of course, the analysis above is extremely simplistic, as fast wind-slow wind interactions, ionization stratifications, and non-spherical geometry all affect the [O III] $\lambda 5007$ 

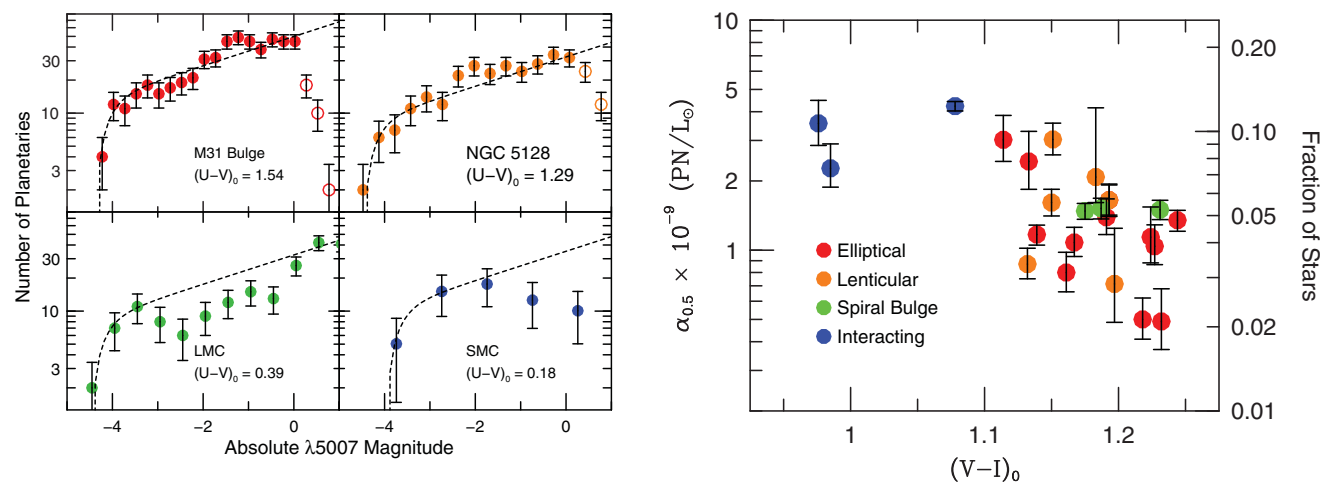

Figure 1. Left: PNLFs from four galaxies with very different colors. The PNLFs of star-forming systems are non-monotonic, while those of systems where current star formation has ceased (even recently) obey the simple Henize \& Westerlund (1963) law. Right: Measurements of the number of PN within $0.5 \mathrm{mag}$ of $M^{*}$ per unit bolometric luminosity for a variety of early-type galaxies. The right-side label represents the fraction of stars evolving into [O III]-bright PN, assuming a

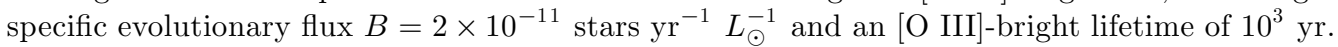

evolution of a planetary nebula. The sophisticated models of Méndez et al. (2008) and Schönberner et al. (2010) include these physical processes, and have been very successful at reproducing the ensemble properties of extragalactic PN. Yet a fundamental puzzle remains about the luminosity function of old stellar populations.

The left-hand panel of Figure 1 shows deep PNLF's for four nearby galaxies. The Magellanic Cloud luminosity functions differ in detail, but both exhibit a non-monotonic behavior consistent with rapid central star evolution. This feature is present in all starforming populations, and argues for the presence of high-mass central stars. In contrast, the PNLFs of M31's bulge (a very red population) and the envelope of NGC 5128 (which has a color close to that of the interarm regions of spirals) both follow the Henize \& Westerlund (1963) exponential. Here, the simplest interpretation is that all the PN are entering the PNLF at the bright end, and then evolving by free nebular expansion.

Yet the simple explanation is impossible. The post-AGB stars produced by old populations should have low-mass cores and be relatively faint. These objects should join the PNLF three or four magnitudes below $M^{*}$ and steepen the luminosity function at those magnitudes. Such behavior has not been observed in any of the deep PNLF surveys of galaxies within $\sim 4 \mathrm{Mpc}$. However, the $\mathrm{PN}$ in the direction of the Galactic bulge do have a steep faint-end slope (Kovacevic et al. 2011), as do the PN candidates in the far halo of M87 (Longobardi et al. 2013). If these two results are confirmed, they would be the first PN-based measurements of an old population's main-sequence turnoff mass.

\section{The Mystery of $M^{*}$}

By far, the most important issue associated with the PNLF concerns the value of $M^{*}$. The bright-end of the PNLF is an excellent standard candle: the brightest PN emit $\sim 630 L_{\odot}$ in [O III] $\lambda 5007$, regardless of the age of the parent population. Since both theory (Dopita et al. 1992; Schönberner et al. 2010) and observations (e.g., Jacoby \& Ciardullo 1999; Kwitter et al. 2012) demonstrate that no more than $\sim 13 \%$ of a postAGB star's luminosity can be reprocessed into [O III] $\lambda 5007$, this implies that the central stars of [O III]-bright PN must at minimum emit $\sim 4800 L_{\odot}$. Even a cursory inspection of evolutionary tracks (e.g., Vassiliadis \& Wood 1994) shows that such luminosities can only 
be produced by relatively high mass $\left(M>0.59 M_{\odot}\right)$ cores. The initial mass-final mass relation (e.g., Kalirai et al. 2008) then implies progenitor masses greater than $1.8 M_{\odot}$.

This result is difficult enough to reconcile with our knowledge of the stellar populations of elliptical galaxies, but the situation is actually much worse than described. During its AGB stage, a star will create a considerable amount of dust. As a result, a typical [O III]bright $\mathrm{PN}$ is enshrouded by $E(B-V) \sim 0.2$ of circumstellar extinction (e.g., Jacoby \& Ciardullo 1999; Herrmann \& Ciardullo 2009; Kwitter et al. 2012). In other words, the true luminosities generated by bright PNe are $\sim 50 \%$ larger than observed. Their central star masses must therefore be greater than $\sim 0.62 M_{\odot}$, implying progenitor masses of over $\sim 2 M_{\odot}$. Populations as young as this $(\sim 1 \mathrm{Gyr})$ are inconsistent with the integrated spectra of old elliptical and lenticular galaxies.

We can estimate that importance of this impossible population by considering the luminosity-specific density of PN in a typical old stellar population. The number of PNe we should expect to see in a galaxy (normalized to its total bolometric luminosity) is simply $\alpha=B \tau f$, where $B$ is the system's luminosity specific stellar evolutionary flux (number of stars evolving into a particular phase of stellar evolution per unit time per unit $\left.L_{\odot}\right), \tau$ is the lifetime of the object, and $f$ is the fraction of stars taking that particular evolutionary path. The first of these quantities is well-known from the theory of stellar

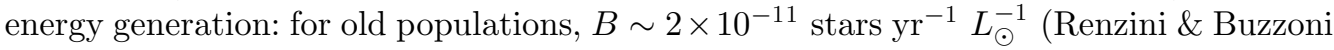
1986). Similarly, the lifetime of a typical [O III] bright PN (i.e., one within $\sim 0.5$ mag of $M^{*}$ ) is also reasonably known, with $\tau \sim 10^{3} \mathrm{yr}$. Finally, as illustrated in the right-hand panel of Figure 1, old stellar populations generally have $\sim 1 \mathrm{PN}$ in the top 0.5 mag of the PNLF for every $10^{9} L_{\odot}$ (Ciardullo et al. 2005). Thus, in old stellar populations, $f \sim 5 \%$ of the main-sequence stars must be evolving into objects with high-mass post-AGB cores.

The evolutionary scenario that produces these high-mass PN central stars is still unclear. In systems which have formed stars in the past Gyr, the formation of post-AGB cores with $M>0.6 M_{\odot}$ can be accomplished by simple single-star stellar evolution. But the presence of high-mass cores in elliptical galaxies demands another evolutionary scenario. While it is well known that binary interactions on the red and asymptotic giant branches can produce PN, this type of evolution usually inhibits the build-up of high mass cores by prematurely ejecting much of the envelope mass into space. This difficulty led Ciardullo et al. (2005) to propose that blue stragglers are the progenitors of the [O III]-bright PN found in old populations. In fact, a color-magnitude diagram of the Ursa Minor dwarf spheroidal galaxy (Carrera et al. 2002) confirms that blue stragglers exist in the right numbers and have the right lifetimes to plausibly be the progenitors of $M^{*} \mathrm{PN}$ (Ciardullo et al. 2005). But much more work is needed to confirm the hypothesis.

Putting aside the progenitor question, the existence of [O III]-bright PN has profound implications for host of astrophysical problems. For example, much of our understanding of galactic stellar populations, both locally and at high redshift, comes from fitting broadband spectral energy distributions (SEDs) to population synthesis models (e.g., Longhetti \& Saracco 2009; Conroy 2013) In this procedure, a proper modeling of the light from AGB stars is critical, since it is this component which most determines a system's total stellar mass. According to current population synthesis models (e.g., Maraston 2005; Bruzual \& Charlot 2003; Bruzual 2007) the old populations in the cores of the Virgo and Fornax Clusters should contain few, if any, AGB cores more massive than $\sim 0.52 M_{\odot}$. Yet the PNLF demonstrates that $M \gtrsim 0.6 M_{\odot}$ cores do exist in these environments, and from a simply scaling of lifetimes, there should be thousands of AGB stars for every [O III]-bright PN. This component is currently being missed in all the SED analyses.

Similarly, the SBF technique is generally considered to be a well-calibrated and accurate method for obtaining the distances to Population II system. Underlying this method 
is the premise that the mean brightness of an old stellar population's red giant stars is a predictable function of galaxy color, and several authors have modeled this behavior (e.g., Cantiello et al. 2003; Cerviño et al. 2008; Conroy \& Gunn 2010). Yet again, these models are ignoring the existence of the most luminous, high-core mass objects.

Progress towards understanding the PNLF's bright-end cutoff is difficult, as it is likely that there is no single evolutionary scenario that creates [O III]-bright planetaries. Nevertheless, it is an area that demands attention, as the uncertainties in the subject propagate into many of the most important topics of astrophysics and cosmology.

\section{References}

Badenes, C., Maoz, D., \& Ciardullo, R. 2015, ApJ (Letters), 804, L25

Bruzual, G. 2007, in A. Vallenari et al. (eds.), ASP Conf. Ser. 374, From Stars to Galaxies: Building the Pieces to Build Up the Universe (San Francisco: ASP), p. 303

Bruzual, G. \& Charlot, S. 2003, MNRAS, 344, 1000

Cantiello, M., Raimondo, G., Brocato, E., \& Capaccioli, M. 2003, AJ, 125, 2783

Carrera, R., Aparicio, A., Martínez-Delgado, D., \& Alonso-García, J. 2002, AJ, 123, 3199

Cerviño, M., Luridiana, V., \& Jamet, L. 2008, A\&A, 491, 693

Ciardullo, R. 2013, in R. de Grijs (ed.), IAU Symp. 289, Advancing the Physics of Cosmic Distances (Cambridge: Cambridge University Press), p. 247

Ciardullo, R., Jacoby, G. H., Ford, H. C., \& Neill, J. D. 1989, ApJ, 339, 53

Ciardullo, R., Jacoby, G. H., \& Ford, H. C. 1989, ApJ, 344, 715

Ciardullo, R., Feldmeier, J. J., Jacoby, G. H., et al. 2002, ApJ, 577, 31

Ciardullo, R., Durrell, P. R., Laychak, M. B., et al. 2004, ApJ, 614, 167

Ciardullo, R., Sigurdsson, S., Feldmeier, J. J., \& Jacoby, G. H. 2005, ApJ, 629, 499

Conroy, C. 2013, ARA\&A A, 51, 393

Conroy, C. \& Gunn, J. E. 2010, ApJ, 712, 833

Dopita, M. A., Jacoby, G. H., \& Vassiliadis, E. 1992, ApJ, 389, 27

Feldmeier, J. J., Ciardullo, R., \& Jacoby, G. H. 1997, ApJ, 479, 231

Ferrarese, L., Mould, J. R., Kennicutt, R. C., Jr., et al. 2000, ApJ, 529, 745

Ford, H. C. 1983, in D.R. Flower (ed.), IAU Symp. 103, Planetary Nebulae (Dordrecht: Reidel), p. 443

Freedman, W. L., Madore, B. F., Gibson, B. K., et al. 2001, ApJ, 553, 47

Henize, K. G. \& Westerlund, B. E. 1963, ApJ, 137, 747

Herrmann, K. A. \& Ciardullo, R. 2009, ApJ, 703, 894

Hui, X., Ford, H. C., Ciardullo, R., \& Jacoby, G. H. 1993, ApJ, 414, 463

Jacoby, G. H., Ciardullo, R., \& Ford, H. C. 1990, ApJ, 356, 332

Jacoby, G. H., Ciardullo, R., \& Walker, A. R. 1990, ApJ, 365, 471

Jacoby, G. H. \& Ciardullo, R. 1999, ApJ, 515, 169

Kalirai, J. S., Hansen, B. M. S., Kelson, D. D., et al. 2008, ApJ, 676, 594

Kovacevic, A. V., Parker, Q. A., Jacoby, G. H., et al. 2011, MNRAS, 414, 860

Kwitter, K. B., Lehman, E. M. M., Balick, B., \& Henry, R. B. C.. 2012, ApJ, 753, 12

Longhetti, M. \& Saracco, P. 2009, MNRAS, 394, 774,

Longobardi, A., Arnaboldi, M., Gerhard, O., et al. 2013, A\&̊A, 558, A42

Maraston, C. 2005, MNRAS, 362, 799

McMillan, R., Ciardullo, R., \& Jacoby, G. H. 1993, ApJ, 416, 62

Méndez, R. H., Riffeser, A., Kudritzki, R.-P., et al. 2001, ApJ, 563, 135

Méndez, R. H., Teodorescu, A. M., Schönberner, D., Jacob, R., \& Steffen, M. 2008, ApJ, 681, 325

Merrett, H. R., Merrifield, M. R., Douglas, N. G., et al. 2006 MNRAS, 369, 120

Renzini, A., \& Buzzoni, A. 1986, in C. Chiosi \& A. Renzini (eds.), Spectral Evolution of Galaxies (Dordrecht: Reidel), p. 195

Schönberner, D., Jacob, R., Sandin, C., \& Steffen, M. 2010, A\&ऽA, 523, A86

Teodorescu, A. M., Méndez, R. H., Bernardi, F., et al. 2010, ApJ, 721, 369

Vassiliadis, E. \& Wood, P. R. 1994, ApJS, 92, 125 